\title{
Zonal mean and tidal dynamics from space: an empirical examination of aliasing and sampling issues
}

\author{
J. M. Forbes ${ }^{1}$, M. Kilpatrick ${ }^{1}$, D. Fritts ${ }^{1}$, A. H. Manson ${ }^{2}$, R. A. Vincent ${ }^{3}$ \\ ${ }^{1}$ University of Colorado, Boulder, Colorado, USA \\ ${ }^{2}$ University of Saskatchewan, Saskatoon, Canada \\ ${ }^{3}$ University of Adelaide, Adelaide, S. Australia
}

Received: 27 September 1996 / Revised: 13 March 1997 / Accepted 15 March 1997

\begin{abstract}
Interpretations of space-based measurements of atmospheric parameters in the mesosphere and thermosphere are complicated by large local-time variations at these altitudes. For this reason, satellite orbits are often preferred which precess through all local times one or more times per season. However, the local-time structure of the atmosphere is inherently non-stationary, which can lead to sampling and aliasing difficulties when attempting to deconvolve the measurements into zonal mean and tidal components. In the present study, hourly radar measurements of mesopause-region winds are used to form a mock data base which can be used to gain insight into implications of the aforementioned problems; the use of actual measurements introduces a realistic element of geophysical temporal variability. Assuming zonal symmetry (i.e., migrating tides superimposed on a zonal mean circulation), the radar measurements are sampled from the satellite perspective for orbital inclinations of $57^{\circ}$ and $70^{\circ}$, and compared to the ground or true perspective. These comparisons provide realistic estimates of the errors to be expected when attempting to derive mean and tidal components from space-based measurements. For both diurnal and semidiurnal components, and the quoted satellite inclinations, acceptable errors $(3-4 \mathrm{~m} / \mathrm{s} \mathrm{rms})$ are obtained for data covering $24 \mathrm{~h}$ local time (i.e., ascending plus descending nodes); the corresponding errors for singlenode data (12 h local-time coverage) are of order $8-11 \mathrm{~m} / \mathrm{s}$, and therefore may not represent reliable estimates of the actual tidal components. There exist certain caveats in connection with the latter conclusion which are discussed.
\end{abstract}

\section{Introduction}

The feasibility of determining zonal mean and atmospheric tidal components of dynamical fields from a local-time precessing space platform is the subject of ongoing discussion and speculation. Several recent papers have appeared which derive tidal oscillations in the neutral wind in the mesosphere/lower thermosphere region (c. 80-120 km; Burrage et al., 1995; Mclandress et al., 1996) using observations from the Upper-Atmosphere Research Satellite (UARS) which has an orbital inclination of $57^{\circ}$ and local-time precession period near 36 days. Similar studies will be performed using data from the TIMED (thermosphere-ionosphere-mesosphere energetics and dynamics) mission, which will have an inclination near $70^{\circ}$ (http://sd-www.jhuapl.edu/ TIMED). The difficulties involved fall into the categories of sampling and aliasing. The sampling issue centers around the inherent geophysical variability of the system within the 24-h local-time precession period of the satellite, which is about 60 days for an inclination $i$ of $70^{\circ}$ (ascending + descending node). In other words, the atmospheric structure ordered in local time does not remain stationary while it is being sampled by the satellite instruments. The aliasing issue is somewhat different. Let us suppose that the tidal components remain stationary, but the zonal mean varies with time. If the atmosphere is sampled from the satellite perspective under this scenario, as slow local-time precession takes place, then the changing zonal mean is perceived as a local-time change, leading to spurious tidal harmonics. In this case we say the mean zonal flow aliases into the tidal components. Evolving tidal structures can alias into the mean, but as we will see, this is a much smaller effect, since the tides tend to cancel out when determining the mean.

The present work takes an empirical approach to the problem, using actual mesopause-region hourly wind observations from the Christmas Island, Hawaii, and 
Saskatoon MF radars to construct a mock satellite data set. These radar data are analyzed from the ground perspective to determine 30-day mean, diurnal, and semidiurnal tidal components for a full year of observations. In a second analysis, sampling of the data proceeds as though orbits (with inclinations of $57^{\circ}$ and $70^{\circ}$ ) are precessed through the data, and mean and tidal components are determined for comparison with the true (ground- based) analyses. In this way, the element of realistic atmospheric variability is introduced, and one can quantitatively assess the validity of deriving zonal mean and tidal components from actual spacebased measurements..

\section{Analysis procedure}

The analysis focuses on a data base consisting of ground-based hourly wind measurements covering 1 year each from the MF radars located at Christmas Island $\left(2^{\circ} \mathrm{N}\right)$, Hawaii $\left(20^{\circ} \mathrm{N}\right)$, and Saskatoon $\left(52^{\circ} \mathrm{N}\right)$. The data base consists of data from these stations during the years 1991, 1990, and 1979, respectively, at the single altitudes of 86,90 , and $90 \mathrm{~km}$. The first step consists of calculating the true or ground perspective mean, diurnal, and semidiurnal wind components from these data using a standard least-squares technique, and stepping through the data so that these dynamical components are calculated once each 30 days (i.e., monthly values). In the exercise performed here, it is then assumed that the wind measurements consist entirely of zonal mean and migrating (sun-synchronous) tidal components, so that the diurnal cycle as perceived from the satellite is independent of longitude. (The calculation of migrating tides from the satellite perspective requires zonally averaging data at fixed local times.) The second step in the analysis consists of precessing satellite orbits $(600 \mathrm{~km}$ circular; $i=57^{\circ}$ and $70^{\circ}$ ) through this mock database, and calculating the corresponding mean, diurnal, and semidiurnal components from the satellite perspective. Ascending node (12-h local-time coverage) and ascending node + descending node (24-h local-time coverage) are considered. [For those unfamiliar with the derivation of tides from satellites, see for example Burrage et al. (1995), McLandress et al. (1996) and references contained therein.) A comparison between the satellite-based and true determinations then provides a reasonable estimate of the actual accuracy with which satellite-based analyses should be able to recover the true dynamical components. A key element of this approach is that actual wind measurements are used to introduce realistic geophysical variability into the satellite sampling procedure.

Table 1 provides the local-time precession periods for the latitudes of each of the radar stations. For each inclination/station combination, data intervals for both the satellite-based and ground- based wind-component determinations were centered on the 15 th day of each month. Although we have performed calculations for satellite inclinations of $30^{\circ}, 57^{\circ}$, and $70^{\circ}$ degrees, only a sampling of the results obtained from the $i=57^{\circ}$ and
Table 1. Precession periods for $24-\mathrm{h}$ local time (ascending \& descending nodes)

\begin{tabular}{lll}
\hline inclination & station & period (days) \\
\hline 30 & Christ. Is. & 26 \\
57 & Christ. Is. & 38 \\
70 & Christ. Is. & 52 \\
30 & Hawaii & 36 \\
57 & Hawaii & 42 \\
70 & Hawaii & 56 \\
70 & Saskatoon & 60 \\
\hline
\end{tabular}

$i=70^{\circ}$ cases are presented here. A more complete summary of the results (Kilpatrick, 1995) may be obtained from the corresponding author upon request.

Attention is drawn to two caveats regarding this procedure. First, forthcoming figures and discussions compare the ground-based or true 30-day (monthly) mean centered on the 15 th day of the month, with the space-based determination also centered on the 15 th of the month, but with a length corresponding to the $12-\mathrm{h}$ or 24-h precession period, as appropriate. The groundbased determinations use data lengths consisting of $24 \mathrm{~h}$ local time in all cases. Therefore, our comparisons provide a measure of how well the space-based determinations reproduce the true monthly climatologies, rather than providing comparisons with respect to exactly equal data lengths. In addition, our assumption that the mock data base is longitude independent is not quite self-consistent with the use (in practice) of satellite data averaged over longitude. Assuming longitude coverage is adequate, the latter tends to remove the effects of planetary waves. However, temporal variability within the mock data base includes the effects of planetary waves, and this variability can in principle affect the space-based determination of tidal components in our calculations. Although this is not a major effect, this inconsistency does tend to exaggerate the errors incurred by space-based determinations of mean winds and tides. Assessments based on our results should bear this in mind.

\section{Sample results for zonal mean wind component}

Figure 1a illustrates zonal mean winds derived from Saskatoon data. (In this and following figures the crosses represent the ground-perspective or true results, and the circles represent the components derived from the satellite perspective.) The top panel of Fig. 1a illustrates the mean northward winds for ascending and descending nodes and a $70^{\circ}$ orbital inclination, and local-time precession period near 60 days. Note that the main trends of the month-to-month variations are captured from the satellite analysis. Differences of $2-5 \mathrm{~m} / \mathrm{s}$ occur during equinoctial months when the true mean meridional wind is small; these differences may be due to the uncertainties introduced by the zonal mean and tidal transition variability characteristic of such periods. The bottom panel illustrates the mean zonal winds derived from a $70^{\circ}$ inclination orbit utilizing 

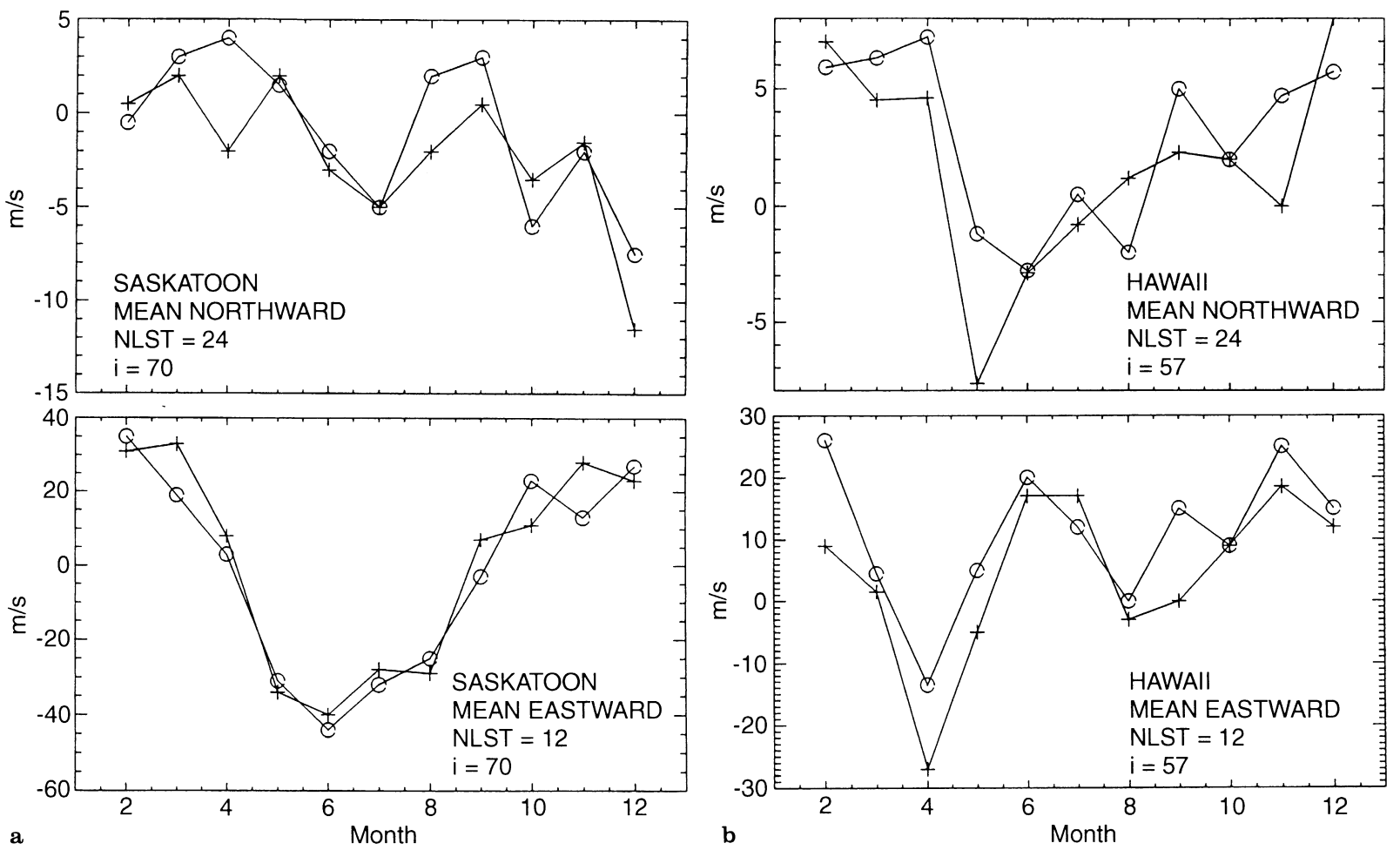

Fig. 1. a Comparison between true (crosses) and space-based (circles) determinations of mean winds at the latitude of Saskatoon $\left(52^{\circ} \mathrm{N}\right)$ for an orbital inclination of $70^{\circ}$. Top: northward wind component for 24 -

h local time coverage. Bottom: eastward wind component for 12-h local-time coverage. b As for a, except with the latitude of Hawaii $\left(20^{\circ} \mathrm{N}\right)$ and an orbital inclination of $57^{\circ}$

ascending node data only ( $12 \mathrm{~h}$ of local time, or 30 days' precession). Because these winds are quite large, the diurnal tide is relatively small at middle latitudes, and the semidiurnal tide is averaged out over $12 \mathrm{~h}$ local time, the monthly variation of mean zonal winds is well reproduced even for ascending node data only.

Figure $1 \mathrm{~b}$ illustrates a similar comparison using measurements of mesopause winds over Hawaii, and assuming a $57^{\circ}$ orbital inclination (precession period of 42 days). Although the 24-h local-time precession period is less, the differences from the true results are somewhat larger than in the previous case. This is because the diurnal tide is large at this location and, especially for the ascending-node- only results, its presence leaks into determination of the mean component.

Figure 2 provides a more quantitative comparison of results for the mean meridional and zonal components. Here the ground- and space-based results are compared in a scatter diagram. In these figures we have combined together the mean wind determinations from Christmas Island, Hawaii and Saskatoon into one data set. Only even months (i.e., February, April, ..., December) are depicted. It is readily apparent that in most cases the satellite-based determinations are in good agreement with the ground-based results (i.e., the data points cluster around the $45^{\circ}$ lines of exact agreement). The correlation coefficients $(R)$ are also quite high $(0.90$ 0.99 ) except for the mean meridional wind determined from $12 \mathrm{~h}$ of data $(R=0.50)$. In this case, the major discrepancies occur for the meridional wind over Hawaii, where it is known that the diurnal component

of the meridional wind can be large. When the diurnal wind is small, or when the null point of the diurnal wind variation occurs near the center of the sampling interval, the space-based determinations of mean meridional wind is valid, even when considering only $12 \mathrm{~h}$ of local-time coverage.

\section{Aliasing issues}

The preceding, relatively good results for the mean component are largely due to the fact that the tidal contributions tend to average out when establishing the mean of all data points during the 24-h or 12-h localtime precession periods. However, when determining the tidal components, any variations in the mean during the course of the precession period are interpreted from the satellite perspective as local-time variations, and hence alias into the tidal determinations. This is illustrated quite well by Fig. 3, wherein the long-term variations in the measured winds (top panel) alias into large diurnal tides which are not present in the true, ground perspective results.

For all the stations listed in Table 1, diagnostic experiments were performed to better understand the sources of aliasing, and how to deal with them. In the first step the data are analyzed as they were in Fig. 3. An example for the semidiurnal tide over Saskatoon is shown in the top panel of Fig. 4. The ground vs. satellite determinations are compared assuming ascending and descending nodes for an orbital inclination of $70^{\circ}$ 

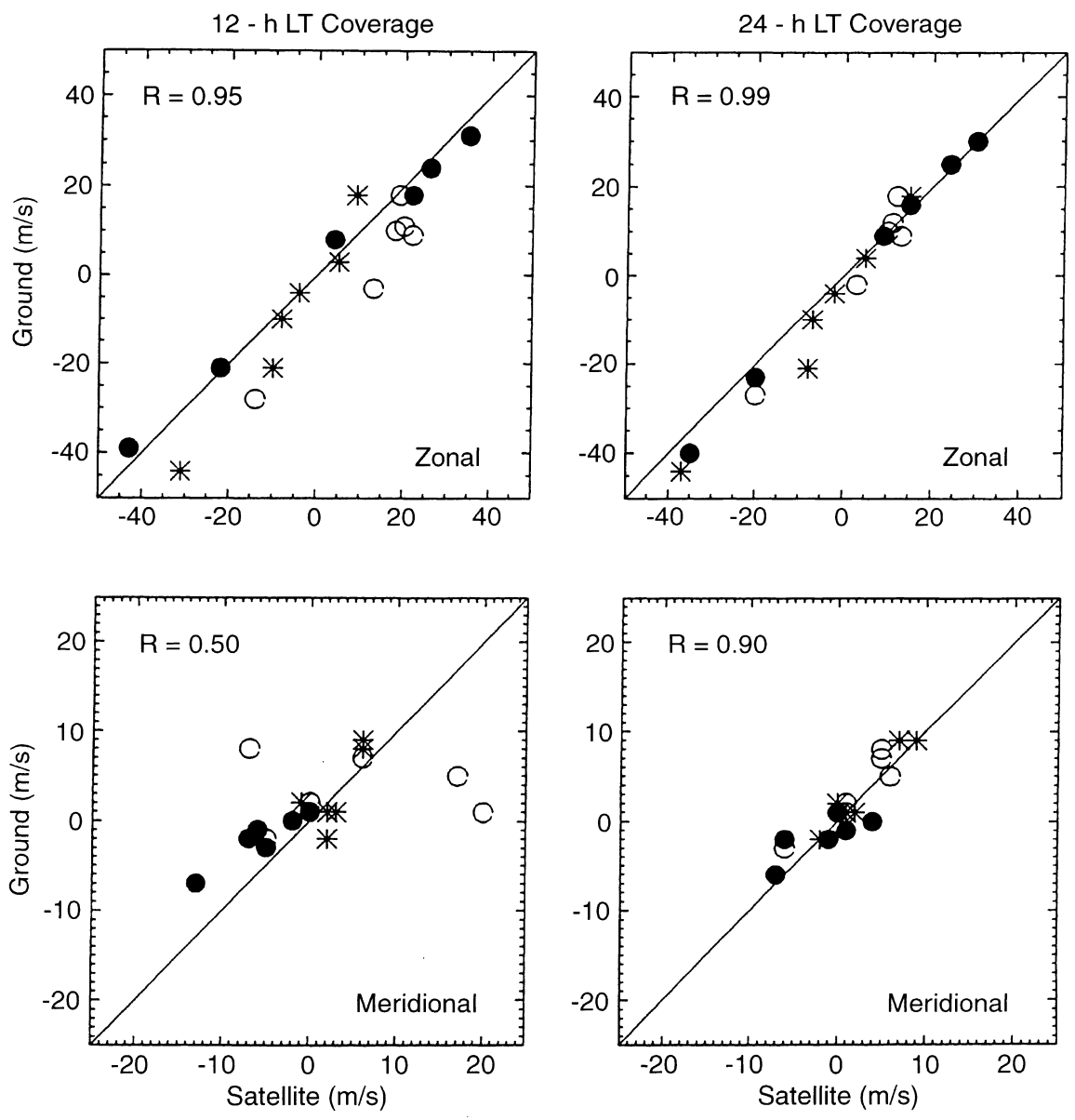

Fig. 2. Comparison between true (groundbased) mean winds and those derived by sampling 1 year of hourly radar measurements (Hawaii, Saskatoon, Christmas Island) near 90$\mathrm{km}$ altitude as though from a satellite at $70^{\circ}$ inclination precessing in local time. The radar observations are assumed to be independent of longitude in these calculations. Determinations for even months only are shown. Left: 12-h local-time coverage; right: 24-h local-time coverage; Top: zonal component; bottom: meridional component. The $R$ values represent linear correlation coefficients and the $45^{\circ}$ diagonals represent the lines of exact agreement between the ground- and space-based determinations

O Hawaii

- Saskatoon

* Christmas I.

(precession period near 60 days). Note that there are significant differences around April/May and September. A second data set is then formed by taking 24-h running averages (slid every hour) of the wind data to remove tidal components. We then repeated the previous analysis procedure (i.e., that used to produce Fig. 3); the middle panel of Fig. 4 illustrates the tides determined from this running-average data set. The tidal components deduced from the satellite perspective on this data set therefore represent the aliasing components due to variations in the mean flow with time.

As a next step, we constructed a third data set by taking hourly differences between the original data set and the running average, thus removing the mean component. The previous analysis was repeated. The resulting mean components deduced from the satellite perspective on this data set (not shown) represent aliasing components due to the tides, which generally turn out to be small (see earlier discussion centering around Fig. 1a, b). The semidiurnal tidal component so derived, illustrated in the bottom panel of Fig. 4, is the tide that can be determined from the data if the mean component is first removed in a simple sliding-fit fashion. Note the improvement over the top panel. This suggests a method of data analysis that might be successful with real satellite data sets. (See McLandress et al., 1996, for a recent example.) This methodology is now used throughout the following section to evaluate the validity of extracting tidal components from the same data base.

\section{Sample results for the tidal components}

Given the issue of dealing with phase as well as amplitude, the method of depiction used in Fig. 2 is also employed for the tidal components. Given a tidal oscillation with frequency $\omega$ and phase $t_{\max }$ (the local time of maximum), the following representation is adopted:

$A \cos \omega\left(t-t_{\max }\right)=a \cos \omega t+b \sin \omega t$.

The $a$ and $b$ coefficients for the space-based and groundbased determinations may now be compared as in Fig. 5. In this figure we consider together, as one data set, tidal determinations corresponding to an inclination of $57^{\circ}$ for both the northward and eastward wind components from Hawaii, and the eastward wind component from Christmas Island. In this depiction only the even-numbered months are considered, since these correspond to independent observations (i.e., no overlap of fitting intervals) for both the $57^{\circ}$ and $70^{\circ}$ inclination cases. Diurnal and semidiurnal components derived from data sets with 24-h and 12-h coverage are shown. As in Fig. 2 the diagonal lines in these plots 


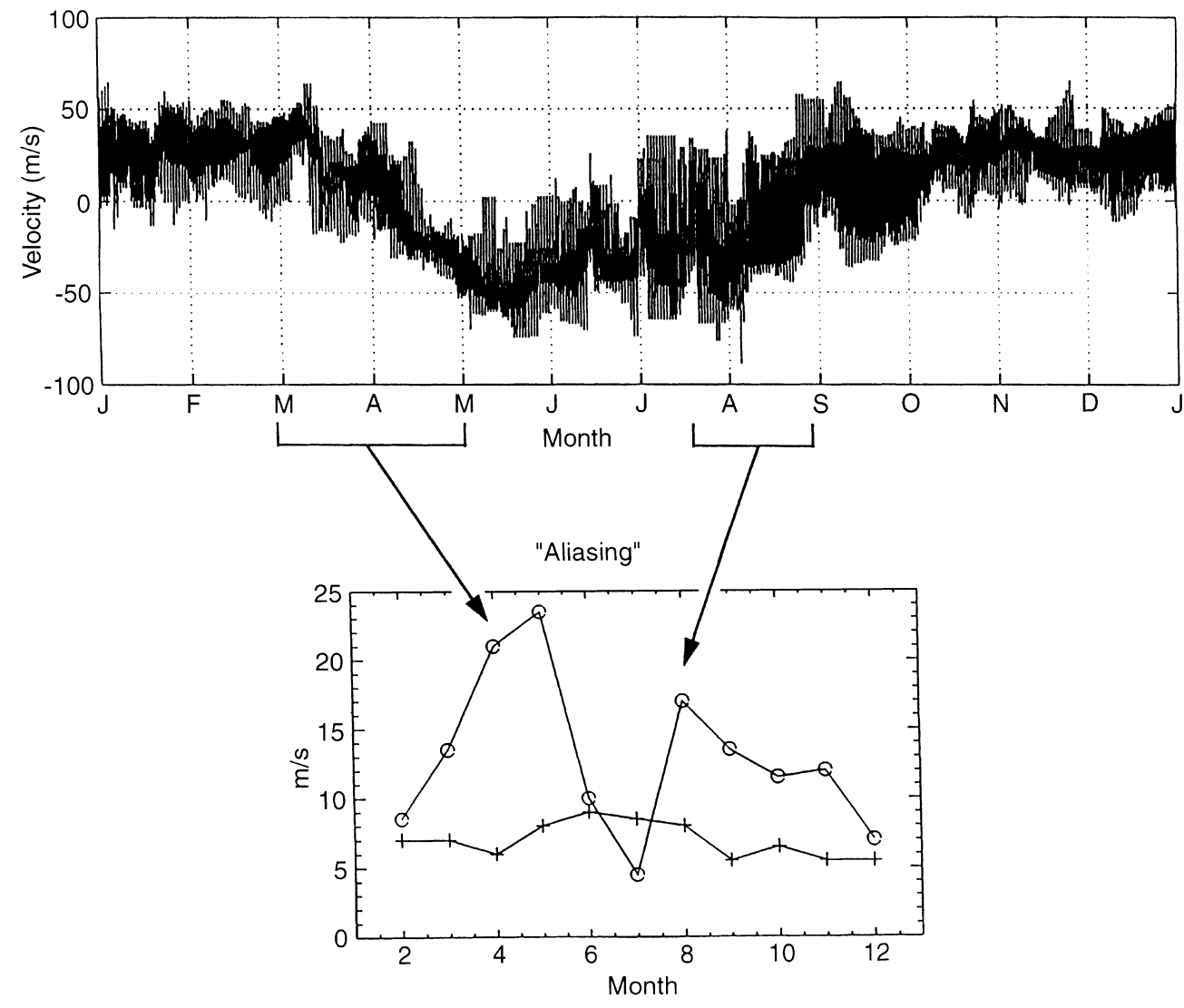

Fig. 3. Illustration of how changes in the mean zonal flow alias into determinations of the diurnal tide using data from a satellite which is precessing slowly in local time. Top: hourly eastward winds over Saskatoon during 1979. Bottom: satellitebased (circles) determinations of the diurnal component of the eastward wind corresponding to an orbital inclination of $70^{\circ}$, compared to the true or ground-based results (crosses). Note how the evolution of the mean circulation around the equinoxes (top) is seen from the satellite perspective as a local-time variation, manifested in the large and erroneous determinations of the diurnal tide (circles, bottom)

represent the hypothetical lines of exact agreement between the space-based and ground-based determinations. The standard deviations of the space-based determinations from these lines, starting at the upper left and moving clockwise, are: 4.48, 10.91, 3.48, and $8.60 \mathrm{~m} / \mathrm{s}$. Also provided in the upper left of each panel are the corresponding linear correlation coefficients

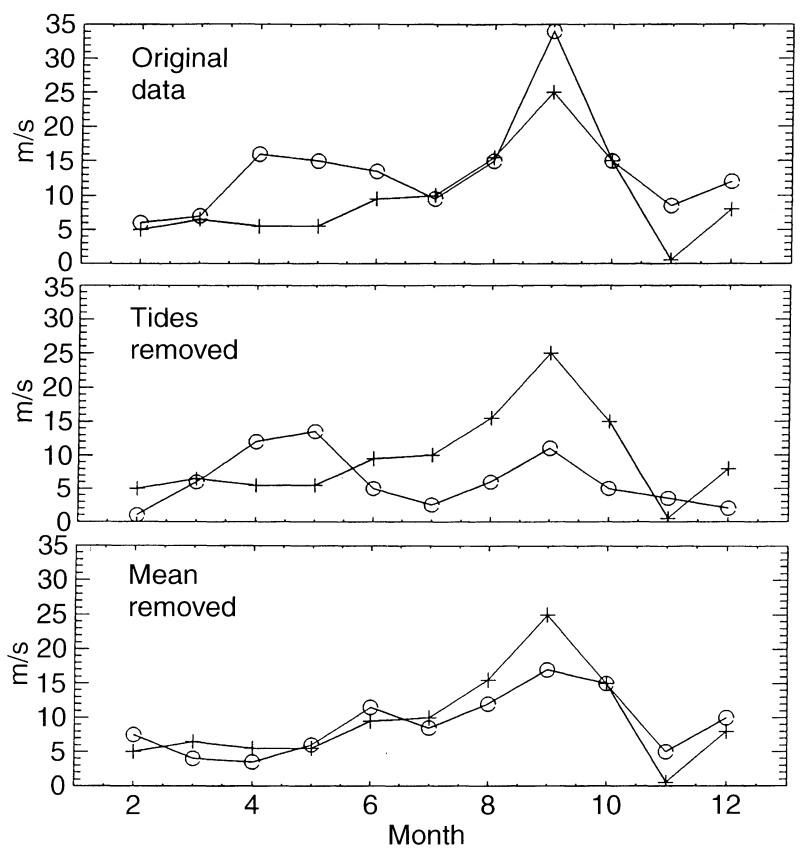

between the two data sets: 0.92, 0.51, 0.80, 0.23. By all measures of comparison, the satellite-based determinations represent reasonable approximations to the true tidal components when $24 \mathrm{~h}$ of local-time data are considered (i.e., ascending plus descending nodes); however, the corresponding determinations for singlenode data (12-h local-time coverage) cannot be considered reliable estimates of the actual tidal components.

A similar comparison is provided in Fig. 6, except for a satellite inclination of $70^{\circ}$ and the combined data set consisting of the eastward and northward wind components over Saskatoon, and the northward wind compo-

Fig. 4. Illustration of how removing the mean component from the data (i.e., using a running mean or low-pass filter) prior to tidal analysis can lead to improved determinations of tidal components from space-based data. All panels correspond to the amplitude of semidiurnal component of eastward wind assuming ascending and descending node data (24-h local-time coverage) at the latitude of Saskatoon $\left(52^{\circ} \mathrm{N}\right)$ for a satellite inclination of $70^{\circ}$. Space-based determinations are given by circles, and true results (same for all panels) are given by crosses. The top panel corresponds to the same data sets and method of analysis used in Figs. 1 and 3. The middle panel results from analysis of the 24-h (local:time) running mean of the data (i.e., the tides are removed) from the satellite perspective; the nonzero tidal amplitudes thus result solely from temporal variations in the mean component aliasing into the tidal determination. The bottom panel illustrates tidal determinations based on the residuals from the 24-h sliding fit, i.e., with the mean removed. Note the improvement over the top-panel results 

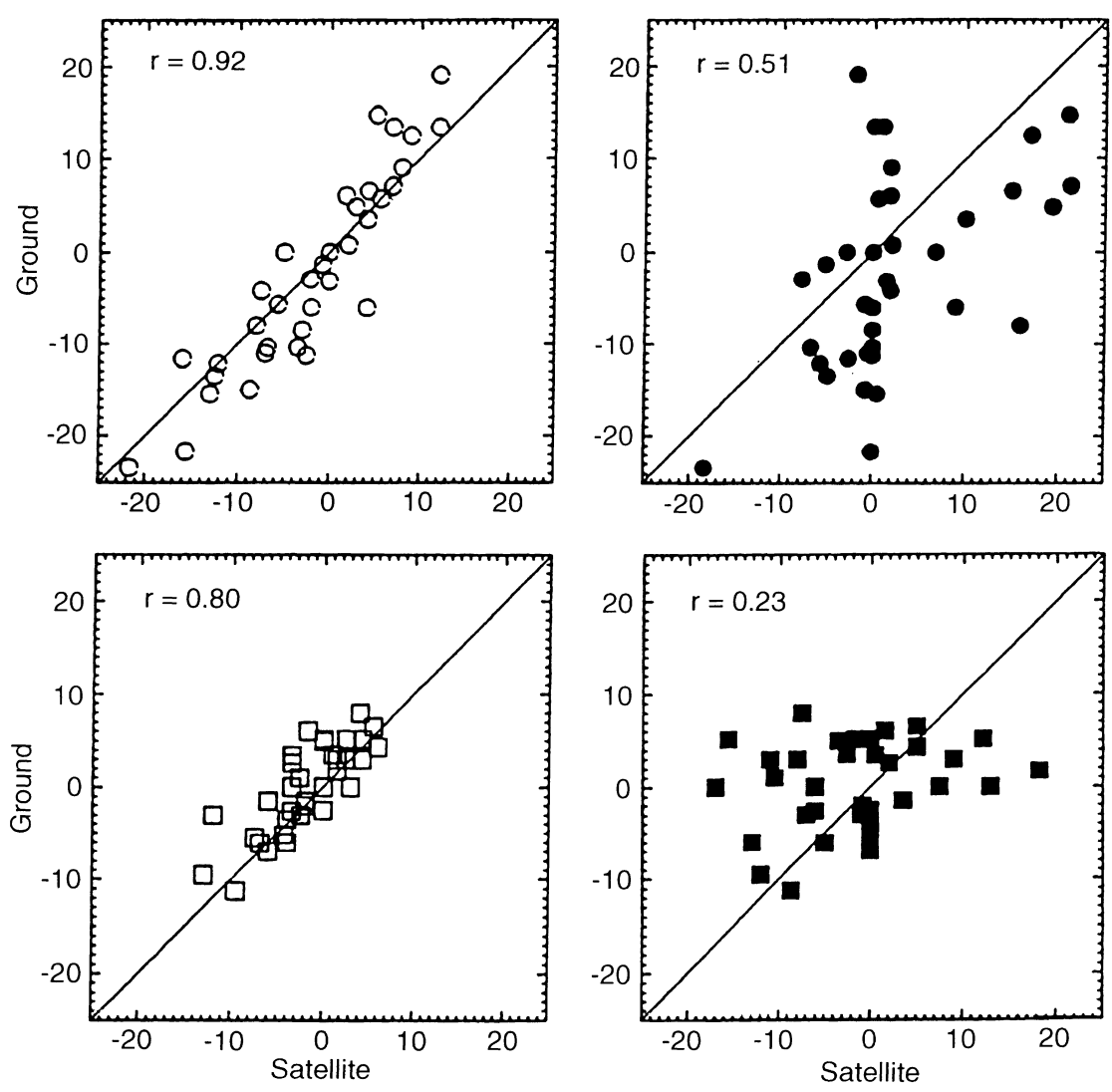

Fig. 5. Comparisons between space-based (abscissa) and ground-tidal (ordinate) determinations, where results are expressed in the form $a \cos \omega t+b \sin \omega t$ where $\omega$ represents the frequency of oscillation, and $a$ and $b$ coefficients are mixed in the presentation. Tidal determinations corresponding to an inclination of $57^{\circ}$ for both the northward and eastward wind components from Hawaii, and the eastward wind component from Christmas Island, taken together as a single data set, are depicted. Open circles: diurnal tide from data covering 24-h local time. Open squares: semidiurnal tide from data covering 24 -h local time. Solid circles and solid squares represent the corresponding tidal components derived from data covering $12 \mathrm{~h}$ local time. The diagonal lines do not represent fits to the data, but correspond to the line of exact agreement. The standard deviations of the space-based determinations from these lines, starting at the upper left and moving clockwise, are: $4.48,10.91,3.48$, and $8.60 \mathrm{~m} / \mathrm{s}$. Also provided in the upper left of each panel are the corresponding linear correlation coefficients between the two data sets: $0.92,0.51,0.80,0.23$
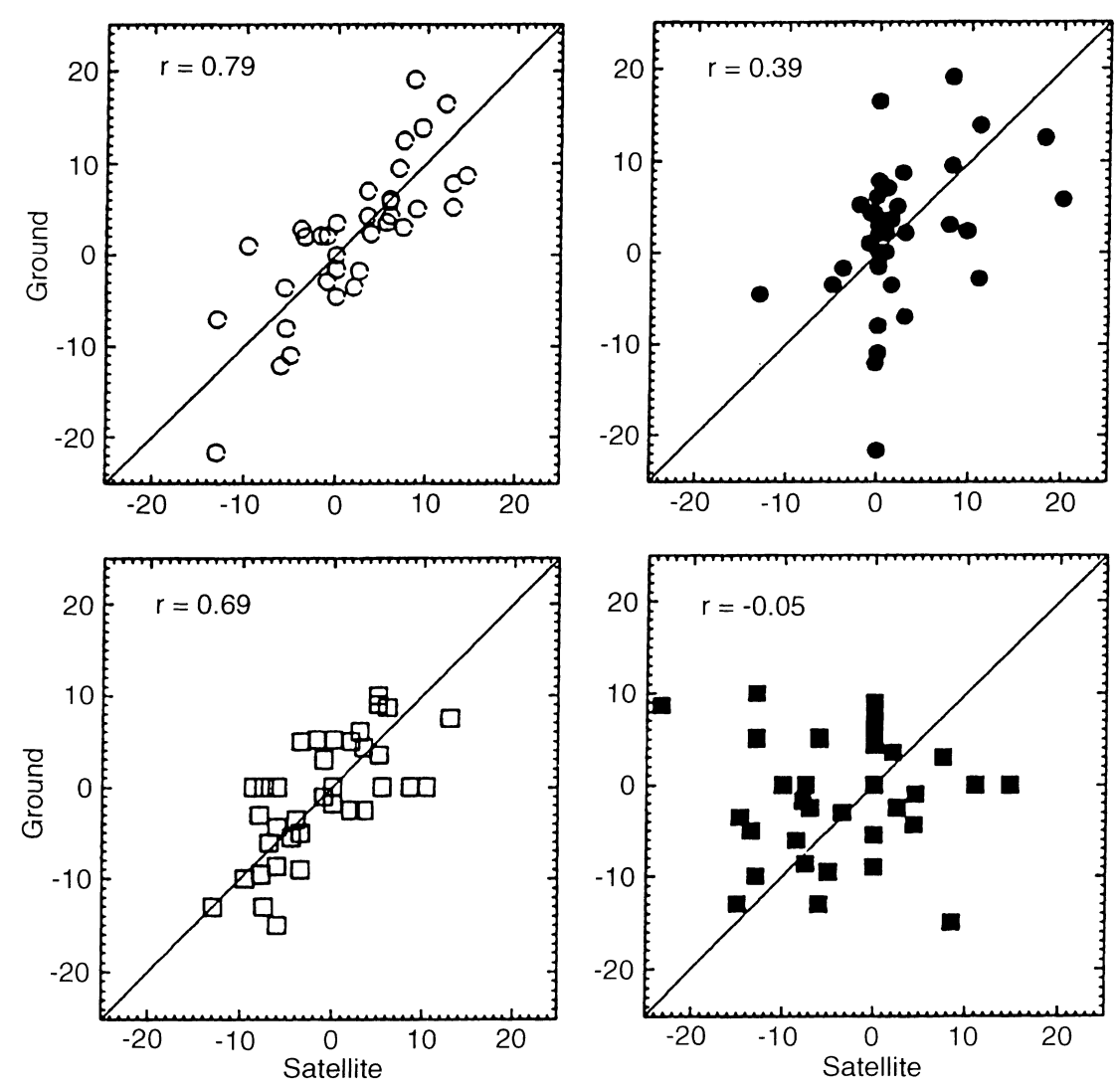

Fig. 6. The same as Fig. 5, except for a satellite inclination of $70^{\circ}$ and the combined data set consisting of the eastward and northward wind components over Saskatoon, and the northward wind component over Hawaii. The standard deviations as defined for Fig. 5 are 5.00, 8.07, 5.11, and $11.33 \mathrm{~m} / \mathrm{s}$, and the respective linear correlation coefficients are $0.79,0.39,0.69,-0.05$ 
nent over Hawaii. The corresponding standard deviations are $5.00,8.07,5.11$, and $11.33 \mathrm{~m} / \mathrm{s}$, and the respective linear correlation coefficients are $0.79,0.39$, $0.69,-0.05$. A slight degradation is noted for this $70^{\circ}$ inclination case as compared with the $57^{\circ}$ inclination results in Fig. 5. However, the basic conclusions already noted regarding the relative reliability of tides determined from both ascending and descending node data, as opposed to single-node data, hold true.

The poor correlations for the semidiurnal tide in Figs. 5 and 6 are due in part to non-stationarity during the fitting interval, and to contamination by the diurnal tide. The latter is particularly true for Fig. 5. When comparisons are made using data from the latitude of Saskatoon, where the diurnal tide is weak, the results are much improved. Moreover, it must be acknowledged that the choice of stations and wind components leading to construction of Figs. 5 and 6 are intended only to provide an overall impression. There are many special cases where the aliasing effects are minimized; i.e., the diurnal meridional wind at tropical latitudes, the semidiurnal meridional wind at middle to high latitudes, etc. It is not possible to address the range of possibilities within the present work, but, again, those who are interested may obtain a complete set of figures (Kilpatrick, 1995) from the corresponding author.

\section{Conclusions}

Space-based determinations of the zonal mean components usually represent faithful reproductions of the true results, even when only 12-h local-time coverage is available. In the latter case, the most serious discrepancies can be expected when the diurnal tidal component is comparatively large and phased such that the diurnal minimum or maximum occurs mid-range within the fitting interval.

The mean component aliases into the tides much more than the tides alias into the mean; essentially, the tidal results average out when determining the mean over the local-time precession intervals.

The preceding suggests that we remove the mean component first (by running mean or low-pass filter; cutoff $=12-\mathrm{h}$ or $24-\mathrm{h}$ precession period), then determine the tidal components.

As noted in Sect. 2, there exist some caveats which may tend to exaggerate our estimates of the aliasing problems encountered in analyzing satellite data. Additional works are underway further to quantify these issues.

Further extensions of the present work are needed to optimize the separation of zonal mean and tidal components from space-based measurements. An important consideration would be inclusion of groundbased measurements at the same altitudes to remove some of the aliasing ambiguities. The synergy between space-based measurements covering a wide range of latitudes and longitudes at relatively constant local times, and that of radar measurements covering all local times at discrete latitudes and longitudes, remains to be explored quantitatively.

It is important to recognize that the present results for $i=70^{\circ}$ underestimate the capabilities of wind measurements from TIMED to determine tides from space. The TIDI instrument (http://sd-www.jhuapl.edu/ TIMED) will make simultaneous measurements at $\pm 45^{\circ}$ and $\pm 135^{\circ}$ to the satellite velocity vector, essentially providing two wind vector measurements separated about $3 \mathrm{~h}$ local time apart along the satellite track. As a bare minimum, this will reduce by $25 \%$ the time needed to precess in local time, from about 60 to 45 days when both day and night measurements are possible. In addition, the distribution of data points in local time afforded by this experimental approach may provide greater improvements in accuracy which remain to be explored in future extensions of the current work.

Acknowledgements. This work was supported by NASA Grant NAG5-2551 to the University of Colorado.

Topical Editor F. Vial thanks R. Liebermann and another referee for their help in evaluating this paper

\section{References}

Burrage, M. D., D. L. Wu, W. R. Skinner, D. A. Ortland, and P. B. Hays, Latitude and seasonal dependence of the semidiurnal tide observed by the high-resolution Doppler imager, J. Geophys. Res., 100, 11313-11321, 1995.

Kilpatrick, M. E., Tides and zonal mean flow from satellite-based remote sensing measurements, M.S. Thesis, Department of Aerospace Engineering Sciences, University of Colorado, Boulder, Colorado, 1995.

McLandress, C., G. G. Shepherd, and B. H. Solheim, Satellite observations of thermospheric tides: results from the wind imaging interferometer on UARS, J. Geophys. Res., 101, 40934114, 1996. 